\title{
Hand, Foot and Mouth Disease (HFMD): An Update
}

\author{
PROBIR KUMAR SARKAR ${ }^{1}$, NITAL KUMAR SARKER ${ }^{2}$, MD. ABU TAYAB ${ }^{3}$
}

\begin{abstract}
Hand, foot, and mouth disease (HFMD) also known as vesicular stomatitis with exanthema, first reported in New Zealand in 1957 is caused by Coxsackie virus A16 (CVA16), human enterovirus 71 (HEV71) and occasionally by other HEV-A serotypes, such as Coxsackie virus $A 6$ and Coxsackie virus A10, are also associated with HFMD and herpangina. While all these viruses can cause mild disease in children, EV71 has been associated with neurological disease and mortality in large outbreaks in the Asia Pacific region over the last decade. It is highly contagious and is spread through direct contact with the mucus, saliva, or feces of an infected person. This is characterized by erythrematous papulo vesicular eruptions over hand, feet, perioral area, knee, buttocks and also intra-orally mostly in children, typically occurs in small epidemics usually during the summer and autumn months. HFMD symptoms are usually mild and resolve on their own in 7 to 10 days. Treatment is symptomatic but good hygiene during and after infection is very important in preventing the spread of the disease. Though only small scale outbreaks have been reported from United States, Europe, Australia Japan and Brazil for the first few decade, since 1997 the disease has conspicuously changed its behavior as noted in different Southeast Asian countries. There was sharp rise in incidence, severity, complications and even fatal outcomes that were almost unseen before that period. There are reports of disease activity in different corners of India since 2004, and the largest outbreak of HFMD occurred in eastern part of India in and around Kolkata in 2007and Bhubaneswar, Odisha in 2009. In recent years there are cases of HFMD have been seen in Bangladesh also. Although of milder degree, continuous progress to affect larger parts of the neighboring may indicate vulnerability of Bangladesh from possible future outbreaks.
\end{abstract}

Key Words: Hand, foot, and mouth disease.

\section{Introduction}

Hand, foot and mouth disease, or HFMD, one of the most distinctive rash syndromes, first reported in New Zealand in 1957 is a contagious illness caused by different viruses. Infants and children younger than 5 years are more likely to get this disease. Older children and adults can also get it. ${ }^{1}$

It is characterized by a brief febrile illness in children andtypical skin rash, with or without mouth ulcers. Typically, the rash is papulovesicular and affects the palms or soles of the feet, or both. In some cases the rash may bemaculopapular without vesicles, and may also involve the buttocks, knees or elbows, particularly

1. Resident Physician (Assistant Professor), Dhaka Shishu (Children) Hospital.

2. Assistant Professor, Dhaka Shishu (Children) Hospital.

3. Assistant Professor, Dhaka Shishu (Children) Hospital.

Correspondence: Dr. Probir Kumar Sarkar, Resident Physician

(Assistant Professor), Dhaka Shishu (Children) Hospital, Dhaka,

Email: tultulprobir@yahoo.com in younger children and infants. ${ }^{2}$ Herpangina or $\mathrm{HA}$ is also characterized by fever and multiple, painful mouth ulcers, predominantly affecting the posterior oral cavity, including the anterior pharyngealfolds, uvula, tonsils and soft palate. In some children, the mouth ulcers can affect otherparts of the mouth, including the buccal mucosa and tongue, with relative sparing of theposterior aspect of the oral cavity. ${ }^{2}$

In practice it is not uncommon for children to complain first of painful oral ulcers beforetypical skin lesions appear over the palms and soles a day or two later. From a clinicalperspective, HFMD and HA could be considered to represent both ends of a spectrum ofmucocutaneous manifestations in a childhood febrile rash syndrome, where herpanginawith isolated oral mucosal involvement is at one end, and HFMD with a combination oforal lesions and skin changes affecting palms and soles at the other. ${ }^{1-3}$ 


\section{Etiology}

The major etiological agents that cause HFMD are the human enteroviruses species A(HEV-A), particularly coxsackievirus A16 (CA16) and enterovirus 71 (EV71). Thesebelong to the genus Enterovirus. Other HEV-Aserotypes, such as Coxsackievirus A6 and Coxsackievirus $A 10$, are also associated withHFMD and herpangina. While all these viruses can cause mild disease in children,EV71 has been associated with neurological disease and mortality in large outbreaks inthe Asia Pacific region over the last decade. ${ }^{4,5}$ Enteroviruses are non-enveloped,small RNA, single-stranded, positive- sense viruses in the picornaviridaefamily. The genus Enterovirus contains a large number of agents that produce a broad range of illnesses and the genus name reflects the importance of the gastrointestinal tract as the primary site of invasion, replication and the source of transmission. ${ }^{6}$

\section{Epidemiology}

Enterovirus infections are common and have a worldwide distribution. Many small and large outbreaks associated with EV71 infection have been reported throughout the world since the early 1970 s. Disease associated with EV71 infection was first described by Schmidt and colleagues in 1974, who reported on 20 patients with CNS disease, including one fatality in California, United States of America, between 1969 and $1972 .{ }^{7}$ In temperate climates there are annual epidemic peaks in spring, summer and fall, although some transmission occurs year-round.Enteroviruses are responsible for $33-65 \%$ of acute febrile illnesses and $55-65 \%$ of hospitalizations for suspected sepsis in infants during the summer and fall in the USA, and $25 \%$ year round. In tropical and semitropical areas, enteroviruses circulate year round. ${ }^{8}$

Humans are the only known reservoir for human enteroviruses. Virus is primarily spread person to person, by the fecal, oral and respiratory routes, and vertically from mother to neonate. Enteroviruses can survive on environmental surfaces, permitting transmission via fomites and also can frequently be isolated from water sources and sewage and can survive for months in wet soil. Transmission occurs within families, daycare centers, schools, playgrounds, summer camps, orphanages, and hospital nurses. If a member of a household is infected, there is $\geq 50 \%$ risk of spread to nonimmune household contacts. ${ }^{1,8}$
The incubation period is typically 3-6 days, except for a 1-3 day incubation period for acute hemorrhagic conjunctivitis. Infected children both symptomatic and asymptomatic, frequently shed cultivable enteroviruses from the respiratory tract for $<1-3$ week, whereas fecal shedding continues up to 7-11 weeks. ${ }^{8}$

Large outbreaks of enterovirus infections have included epidemics of echovirus meningitis in numerous countries, epidemics of hand-foot-and-mouth disease with severe central nervous system (CNS) and/or cardiopulmonary disease in young children due to enterovirus 71 in Asia and Australia and outbreaks of acute hemorrhagic conjunctivitis due to enterovirus 70 , coxsackievirus A24, and coxsackievirus A24 variant in tropical and temperate regions. China reported yearly outbreaks from January to July since 2009. The number of cases reported was 115,000 cases in $2009,70,756$ in 2010, 1,654,866in 2010, 1,340,259 in 2011 and 1,520,274 cases in 2012. There were 50 deaths in 2009, 537 in 2010, 437 in 2011 and 431 deaths were reported in 2012.9,10

\section{Clinical Features}

Hand, foot and mouth disease is usually a mild illness, with or without low-grade fever. The oropharynx becomes inflamed and contains scattered vesicles on the tongue, buccal mucosa, posterior pharynx, palate, gingival, and/or lips. These may ulcerate, leaving 4 to $8 \mathrm{~mm}$ shallow lesions with surrounding erythrema.Maculopapular, vesicular and/or pastular lesions may occur on the hands and fingers, feet and buttocks and groin. The hands are more commonly involved than the feet, usually mildly tender and occur more commonly on the dorsal surfaces but frequently also on palms and soles and resolve in about one week. HFMD caused by enterovirus 71 is frequently more severe than coxackievirus A16 disease, with high rates of neurologic and cardiopulmonary involvement, including brainstem encephalomyelitis, neurogenic pulmonary edema, pulmonary hemorrhage, shock and rapid death, especially in young children. ${ }^{1,8}$

Herpangina is characterized by sudden onset of fever, sore throat, dysphagia, and lesions in the posterior pharynx. Blisters and sores in the mouth can make eating and swallowing painful, so children may not want to eat or drink. Headache and backache may occur in older children, and vomotting and abdominal pain occur in $25 \%$ cases. Characteristic lesions, present on the anterior tonsillar pillars, soft palate, uvula, tonsils, posterior pharyngeal wall, and 
occasionally, the posterior buccal surfaces, are discrete 1-2 $\mathrm{mm}$ vesicles and ulcers that enlarge over 2 to 3 days to $3-4 \mathrm{~mm}$ and are surrounded by erythrematous rings that vary in size up to $10 \mathrm{~mm}$. Most cases are mild, fever generally lasts 1-4 days and resolution of symptoms occurs in 3-7 days. ${ }^{1,8}$

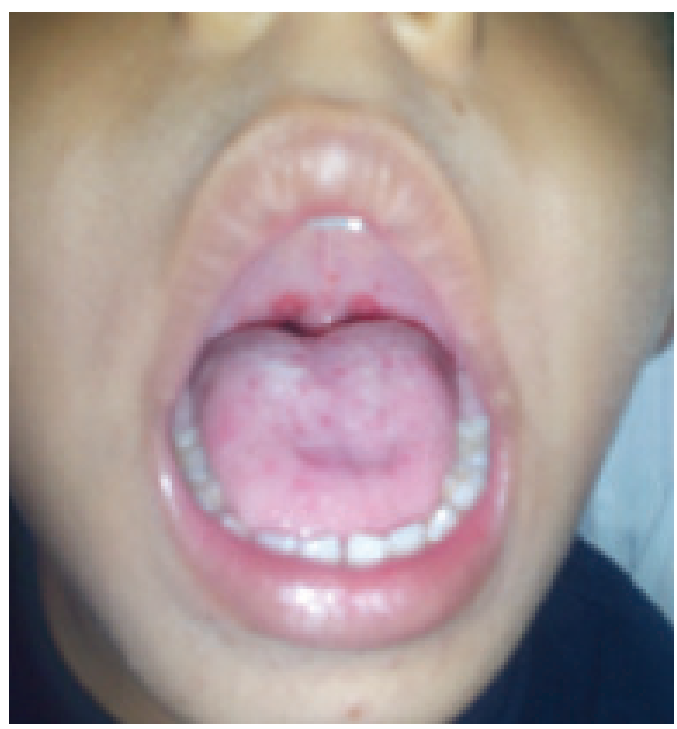

Fig.-1: Herpingina

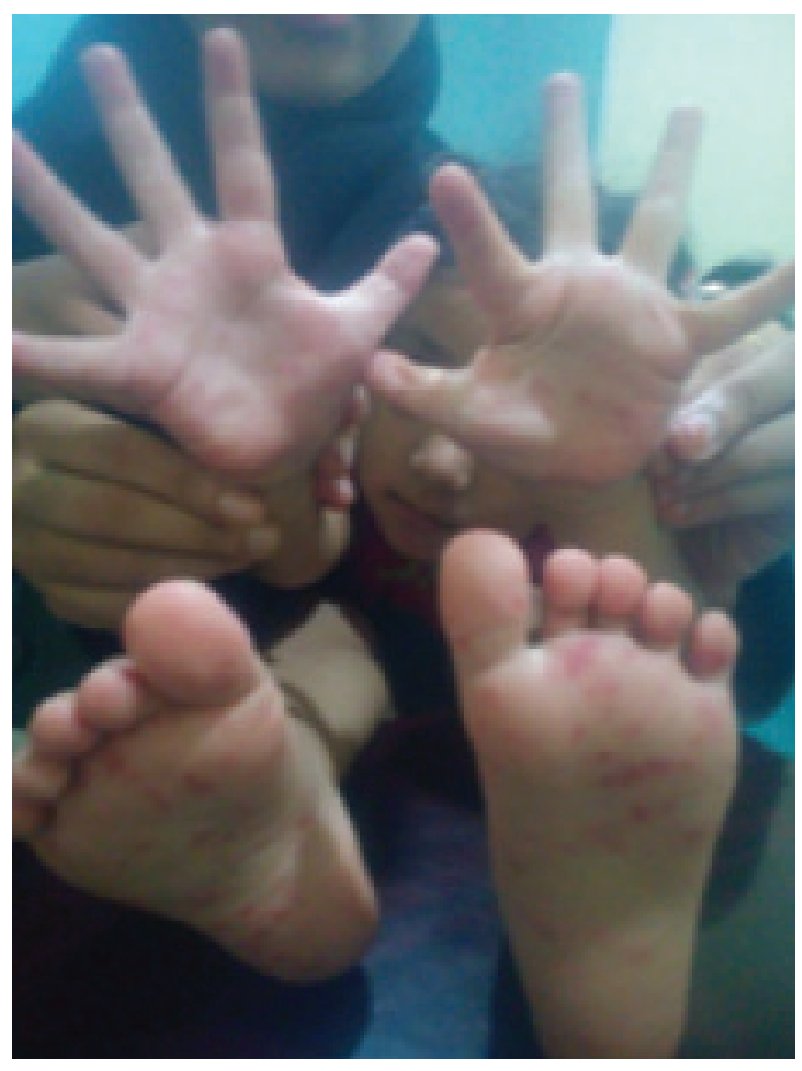

Fig.-2: Rash on palms and soles

\section{Differential Diagnosis}

The differential diagnoses for HFMD include herpetic gingivostomatitis, aphthousstomatitis, scabies infestation, chickenpox (varicella), measles and rubella. In herpeticgingivostomatitis, patients are usually febrile and look toxic. They may have gingivalerythema, swelling or bleeding, and associated cervical lymphadenopathy. There maybe circumoral ulcers or vesicles without extremity involvement. Aphthous stomatitisis characterized by larger, ulcerative lesions of the lips, tongue and buccal mucosa thatare exquisitely painful. It most commonly affects older children and adults, can havemultiple recurrences, and is generally not associated with constitutional symptoms.Scabies infestation may sometimes be confused with HFMD because it also causespustules, vesicles or nodular lesions over the hands and feet. An intense itch and interdigitalspace involvement are useful clinical clues to parasitic infestation. In contrastto HFMD, varicellar lesions are centrifugal in distribution and involve a larger skinarea, including the scalp, but spare the palms and soles. The varicellar lesions healby formation of crusts, while vesicles of HFMD resolve by reabsorption of vesicularfluid. Besides generalized maculopapular rash, children with a typical measles infectionoften present with cough, coryza and conjunctivitis, and koplik spots may be found onexamination of the mouth. The skin rash in rubella has centripetal distribution andoccipital lymphadenopathy. ${ }^{1}$

\section{Diagnosis}

Diagnosis is usually clinical and based on the patient's age, symptoms, and type and location of rash or sores. Generally, a doctor does not need a test to diagnose HFMD. Sometimes, he or she may take a throat swab or collect a sample of blister fluid or stool to test what kind of enterovirus is causing illness.

\section{Treatment}

Medications are usually not needed as HFMD is a viral disease that typically gets better on its own. Currently, there is no specific treatment for hand, foot and mouth disease. However, some things can be done to relieve symptoms, such as

- Taking over-the-counter medications to relieve pain and fever (Caution: Aspirin should not be given to children).

- Using mouthwashes or sprays that numb mouth pain. 
- If the baby cannot swallow and becomes dehydrated, intravenous fluid may be needed.

Mild HFMD cases only need symptomatic treatment. Treatment of fever and relief of symptoms,

adequate hydration and rest are important. Parents and care takers should be educated on hygieneand measures that they should take to prevent transmission to other children. ${ }^{1,8}$

\section{Complications}

Complications from the viral infections that cause HFMD are rare, but require immediate medicaltreatment if present. HFMD infections caused by Enterovirus 71 tend to be more severe and are morelikely to have neurologic or cardiac complications including death than infections caused byCoxsackievirus A16. ${ }^{11}$ Viral or aseptic meningitis can occur with HFMD in rare cases and ischaracterized by fever, headache, stiff neck, or back pain. The condition is usually mild and clearswithout treatment however, hospitalization for a short time may be needed. Other serious complicationsof HFMD include encephalitis (swelling of the brain), or flaccid paralysis in rare circumstances. ${ }^{11,12}$ Fingernail and toenail loss have been reported in children 4-8 weeks after having HFMD. Therelationship between HFMD and the reported nail loss is unclear however, it is temporary and nailgrowth resumes without treatment. ${ }^{13}$

\section{Prevention}

There is no vaccine to protect against the viruses that cause hand, foot, and mouth disease.

A person can lower their risk of being infected by

- Washing hands often with soap and water, especially after changing diapers and using thetoilet. Cleaning and disinfecting frequently touched surfaces and soiled items, includingtoys.

- Avoiding close contact such as kissing, hugging, or sharing eating utensils or cups withpeople with hand, foot, and mouth disease.

\section{If the outbreak occurs in primary schools}

- Principals, teachers and supervisors shall be alerted to look out for children with fever,rash / blisters on palms and soles and to isolate them immediately. Screening beforecoming to class is recommended.
- Ensure that the infected children remain away from the institution for at least ten daysafter onset of symptoms and must be certified free from infection by a registered medicalpractitioner prior to returning to school.

- Health education to the students on the disease, mode of transmission, importance ofgood personal hygiene.

- If closure is necessary, just closed the affected class. Closure of the whole school isunnecessary as HFMD in older children is usually very mild and so far no complicationhas been documented from this age group.

\section{Hand, Foot and Mouth Disease: Bangladesh Perspective}

The first major outbreak of HFMD occurred in Sarawak, Malaysia in 1997 in the Asia Pacific region. ${ }^{14}$ Though there are reports of disease activity in different corners of India since 2004, the largest outbreak of HFMD occurred in eastern part of India in 2007, where about 38 cases of HFMD in and around Kolkata was reported. ${ }^{15}$ Seventy eight casesd of HFMD mostly children from 5-14 years were detected between September 7 and November 6, 2009 in Bhubaneswar, Odisha. ${ }^{16}$ Although of milder degree, continuous progress to affect larger parts of the neighboring may indicate vulnerability of Bangladesh from possible future outbreaks. Though there is no national data on HFMD in Bangladesh, potential human infecting enterovirus strains are present in non-human primates and an enterovirus outbreak of conjunctivitis where about 70 cases was reported in 1981. ${ }^{17,18}$

\section{Conclusion}

In contrast to poliomyelitis, another enteroviral disease renowned for its significant neurological complications, HFMD has been considered to be a benign disease of self limiting nature. For this reason this has got less attention from the medical fraternity, researchers, public health department and policy makers. Following the near complete eradication of poliovirus, HEV71, the non-polio enterovirus may become the greatest threat to cause significant neurological complications. This is evident from the non-availability of effective vaccines or stringent preventive policy. There is insufficient level of awareness among the practitioners. Now with many fatal attacks in different Southeast Asian countries, it has become a cause of concern. 


\section{References}

1. Robinson CR, Doane FW, Rhodes AJ. Report of an outbreak of febrile illness with pharyngeal lesions and exanthema: Toronto, summer 1957; isolation of group A Coxackie virus. Can Med Assoc J. 1958; 79: 615-21.

2. Ooi MH. Clinical features, diagnosis and management of human enterovirus 71 infection.Lancet Neurology.2010; 9:1097-1105.

3. World Health Organization. Enterovirus type 71 surveillance, 1979.

4. McMinn P. Phylogenetic analysis of enterovirus 71 strains isolated during linked epidemics in Malaysia, Singapore, and Western Australia. Journal of Virology. 2001;75:7732-38.

5. Bible JM. Genetic evolution of enterovirus 71: epidemiological and pathological implications. Reviews in Medical Virology. 2007;17:371-79.

6. Oberste MS. Improved molecular identification of enteroviruses by RT-PCR and amplicon sequencing. Journal of Clinical Virology.2003; 26:375-77.

7. Schmidt NJ, Lennette EH, Ho HH. An apparently new enterovirus isolated from patients with disease of the central nervous system. Journal of Infectious Diseases. 1974;129:304-09.

8. Kliegman RM, Stanton BF, St. Geme JW, Schor NF, Behrman RE. Nelson Textbook of Pediatrics. $19^{\text {th }}$ ed. Elsevier; 2013. p. 1088-1092.

9. Emerging disease surveillance and response. Hand, Foot and Mouth Disease. World Health Organization 2013. Retrived 16 October 2013.
10. Hand, foot and mouth disease.https:// en.wikipedia.org/wiki/hand,_foot_and_ mouth_disease

11. Sharma N. Hand, foot and mouth disease: current scenario and Indian perspective. Indian Journal of Dermatology, Venereology andLeprology. 2013;79: 165-75.

12. Huang CC, Liu CC, Chang YC, Chen CY, Wang ST. Neurologic complications in children with enterovirus 71 infection. The New England Journal of Medicine. 1999;341: 936-42.

13. Hand, Foot and Mouth Disease. Complications. Centers for Disease Control and Prevention. 2011. Retrieved 18 October 2013.

14. Podin Y, Gias EL, Ong F, Leong YW, Yee SF, Yusof MA, et al. Sentinel surveillance for human enterovirus 71 in Sarwak, Malaysia: Lessons from the first 7 years. BMC Public Health. 2006; 6:180.

15. Sarma N, Sarkar A, Mukherjee A, Ghosh A, Dhar $S$, Malakar R. Epidemic of hand, foot and mouth disease in West Bengal, India in August 2007: A multicentric study. Indian J dermatol. 2009;54: 26-30.

16. Bikash R K, Bhagirathi D, Shantanu K K. An outbreak of Hand, Foot and Mouth Disease in Bhubaneswar, Odisha. Indian Pediatr. 2013; 50: $139-42$.

17. An outbreak of Enterovirus conjunctivitis in Bangladesh.Trans R. Soc Trop Med Hyg. 1983;77:217-218.

18. www.gideononline.com accessed on 11 February 2015. 